\title{
Assessment of Intracranial Collaterals on CT Angiography in Anterior Circulation Acute Ischemic Stroke
}

\author{
L.L.L. Yeo, P. Paliwal, H.L. Teoh, R.C. Seet, B.P. Chan, E. Ting, N. Venketasubramanian, W.K. Leow, B. Wakerley, Y. Kusama,
}

R. Rathakrishnan, and V.K. Sharma

\begin{abstract}
BACKGROUND AND PURPOSE: Intracranial collaterals influence the prognosis of patients treated with intravenous tissue plasminogen activator in acute anterior circulation ischemic stroke. We compared the methods of scoring collaterals on pre-tPA brain CT angiography for predicting functional outcomes in acute anterior circulation ischemic stroke.
\end{abstract}

MATERIALS AND METHODS: Two hundred consecutive patients with acute anterior circulation ischemic stroke treated with IV-tPA during 2010-2012 were included. Two independent neuroradiologists evaluated intracranial collaterals by using the Miteff system, Maas system, the modified Tan scale, and the Alberta Stroke Program Early CT Score 20-point methodology. Good and extremely poor outcomes at 3 months were defined by modified Rankin Scale scores of $0-1$ and 5-6 points, respectively.

RESULTS: Factors associated with good outcome on univariable analysis were younger age, female sex, hypertension, diabetes mellitus, atrial fibrillation, small infarct core (ASPECTS $\geq 8$ ), vessel recanalization, lower pre-tPA NIHSS scores, and good collaterals according to Tan methodology, ASPECTS methodology, and Miteff methodology. On multivariable logistic regression, only lower NIHSS scores (OR, 1.186 per point; 95\% Cl, 1.079-1.302; $P=.001$ ), recanalization (OR, 5.599; 95\% Cl, 1.560-20.010; $P=.008$ ), and good collaterals by the Miteff method (OR, 3.341; 95\% Cl, 1.203-5.099; $P=.014$ ) were independent predictors of good outcome. Poor collaterals by the Miteff system (OR, 2.592; 95\% Cl, 1.113-6.038; $P=.027$ ), Maas system (OR, 2.580; 95\% Cl, 1.075-6.187; $P=.034$ ), and ASPECTS method $\leq 5$ points (OR, $2.685 ; 95 \%$ $\mathrm{Cl}, 1.156-6.237 ; P=.022)$ were independent predictors of extremely poor outcomes.

CONCLUSIONS: Only the Miteff scoring system for intracranial collaterals is reliable for predicting favorable outcome in thrombolyzed acute anterior circulation ischemic stroke. However, poor outcomes can be predicted by most of the existing methods of scoring intracranial collaterals.

ABBREVIATION: AAIS = acute anterior circulation ischemic stroke

A cute occlusion of an intracranial artery is responsible for the clinical manifestations in acute anterior circulation ischemic stroke (AAIS), and rapid dissolution of the offending arterial thrombi often leads to dramatic clinical recovery. ${ }^{1}$ Therefore,

Received June 17, 2014; accepted after revision July 27.

From the Division of Neurology, Department of Medicine (L.L.L.Y., P.P., H.L.T., R.C.S., B.P.C., N.V., B.W., Y.K., R.R., V.K.S.), and Department of Diagnostic Imaging (E.T.), National University Health System, Singapore; and Department of Computer Science (W.K.L.) and Yong Loo Lin School of Medicine (R.C.S., V.K.S.), National University of Singapore, Singapore.

This work was supported by the National Medical Research Council, Singapore, grant No. CNIG12nov001.

Please address correspondence to Leonard Leong Litt Yeo, MBBS, MRCP, Division of Neurology, Department of Medicine, National University Health System, Singapore, One E Kent Ridge Rd, Singapore 119228; e-mail: leonard_Il_yeo@nuhs.edu.sg

O-. Indicates open access to non-subscribers at www.ajnr.org

三 Indicates article with supplemental on-line table.

http://dx.doi.org/10.3174/ajnr.A4117 achieving timely recanalization remains the main aim of acute stroke care. $^{2}$

Although the parenchymal ischemic injury is restricted to the vascular territories of the index artery, neuronal damage and its clinical manifestations are not uniform in patients with AAIS, especially during the first few hours after an acute intracranial occlusion. These phenomena are observed due to, at least to some extent, variations in the quality and quantity of collateral perfusion. ${ }^{3}$ Detailed imaging studies have shown that progression to complete infarction is highly variable and the process might take many hours or even days to complete. ${ }^{4}$

Irrespective of the mechanism of ischemia, various collateral pathways are recruited to limit ischemic injury to the brain..$^{5-7}$ The wide variations in the clinical manifestations and the rates and extent of neurologic recovery in patients with AAIS may be attributable to these collateral pathways, comprising an intact circle of Willis, and the state of leptomeningeal collaterals connect- 


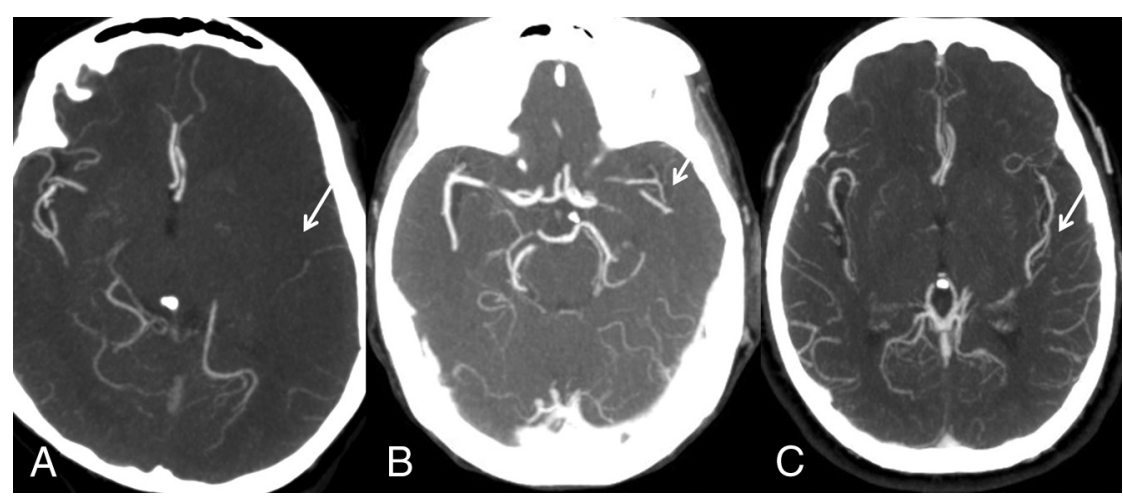

FIG 1. Miteff system. A, Contrast opacification is seen merely in the distal superficial branches. $B$, Vessels can be seen at the Sylvian fissure. C, Major vessels are reconstituted distal to the occlusion.

ing vascular territories. An acute intracranial occlusion produces an area of ischemic penumbra, the extent being dependent on residual and the collateral blood flow. ${ }^{8-10}$ Intravenously administered tissue plasminogen activator, the only approved drug therapy in AAIS, can salvage this penumbra if arterial recanalization is achieved in time. CT angiography of the brain is frequently performed for AAIS. Various methods have been described for the assessment of intracranial collaterals on CTA in patients with AAIS. However, there is no consensus on the best method to evaluate and grade collaterals. Current methods of assessment of collaterals are largely qualitative or semiquantitative, without any clear indication of the superiority of one technique over another. We compared various methods of scoring collaterals on the pretreatment CTA of the brain to determine their value in the prediction of functional outcome in our patients with AAIS treated with IV-tPA.

Ethics approval for this project was obtained from the institutional review board.

\section{MATERIALS AND METHODS}

We identified patients from our acute stroke data base; consecutive patients with AAIS treated with IV-tPA from January 2010 to December 2012 were considered for the study. Inclusion criteria were patients with AAIS who had a pretreatment CTA performed and then underwent IV-tPA. Patients who were pregnant, had poor-quality CTA scans, had only distal anterior cerebral artery occlusion, or were unable to come for follow-up appointments were excluded from the study. If consent could not be obtained from the patient or relatives, the patients were also excluded. We collected data for demographic characteristics and various vascular risk factors such as hypertension, diabetes mellitus types 1 and 2, dyslipidemia, atrial fibrillation, and smoking. Systemic blood pressure values were recorded for all patients at presentation, and levels were maintained during the first few days according to the recommended guidelines. ${ }^{11}$ AAIS was classified into various subtypes by using the Trial of Org 10172 in Acute Stroke Treatment classification on the basis of the etiopathologic mechanisms. ${ }^{12}$ National Institutes of Health Stroke Scale scores were recorded for all patients by credentialed neurologists before the IVtPA bolus and at 2 and 24 hours after treatment initiation. Functional outcome was assessed by modified Rankin Scale at 3 months. Good and poor functional outcomes were defined by
mRS scores of $0-1$ and 2-6, respectively. Extremely poor outcomes were defined by mRS scores of 5-6.

In addition to the noncontrast CT scan of the brain, high-resolution CTA was performed in all patients. In patients with no contraindication for the radiocontrast injection (specifically, contrast allergy or serum creatinine levels of $>110$ mol/L), high-resolution CTA was performed on a 64-section multidetector helical scanner (Brilliance; Philips Healthcare, Best, the Netherlands), and images were acquired with a $70-\mathrm{mL}$ bolus injection of contrast at $3 \mathrm{~mL} / \mathrm{s}$ followed by 30 $\mathrm{mL}$ of saline at $3 \mathrm{~mL} / \mathrm{s}$ into the antecubital vein. Scanning was triggered by using bolus tracking, with the region of interest placed in the posterior aortic arch with the trigger threshold set at $150 \mathrm{HU}$. Scan parameters at our institution were the following: section thickness, $1 \mathrm{~mm}$; no section gap; FOV, $200 \mathrm{~mm}$; matrix, $512 \times 512$; and 230-250 mAs. Coverage was from the base of the skull to the vertex, and the source images were reformatted into 3-mm-thick axial, coronal, and sagittal projections. MIPs were routinely provided as part of CTA; no 3D reconstructions were performed.

All CTA images were anonymized and reviewed at the same workstation, independently by 2 experienced neuroradiologists (E.T. and G.B.) blinded to the patient clinical information and results. Each CTA study was evaluated for intracranial collaterals according to the 4 following predefined criteria:

Miteff System. The system of Miteff et $\mathrm{al}^{13}$ is a 3-point score that grades middle cerebral artery collateral branches with respect to the Sylvain fissure and can be performed rapidly. The grades assigned are the following: 3 (if the vessels are reconstituted distal to the occlusion), 2 (vessels can be seen at the Sylvian fissure), or 1 (when the contrast opacification is merely seen in the distal superficial branches) (Fig 1).

Maas System. The system of Mass et $\mathrm{al}^{14}$ is a 5-point score that compares collaterals on the affected hemisphere against those on the unaffected side. It uses the Sylvian fissure vessels or leptomeningeal collaterals as internal controls. The score ranges are 5 (exuberant), 4 (more than those on the contralateral side), 3 (equal to those on the contralateral side), 2 (less than those on the contralateral side), and 1 (no vessel opacification) (Fig 2).

Modified Tan Scale. The modified scale of Tan et $\mathrm{al}^{15}$ is the simplest system that classifies the collaterals as "good" if seen in $\geq 50 \%$ of the MCA territory and "poor" when they are seen in $<50 \%$ of the territory. This system allows a rapid assessment and is less prone to differences in opinion (Fig 3).

Alberta Stroke Program Early CT Score Methodology using a 20Point Grading Scale. Collaterals are scored in regions corresponding to the ASPECTS system. Lenticulostriate arteries in the basal ganglia arising from retrograde-filling MCAs distal to an occlusion are included in the scoring. The system scores the extent of contrast opacification in arteries distal to the occlusion (0, 




FIG 2. Maas system. $A$, No vessel opacification. $B$, Opacification less than that on the contralateral side. Opacification equal to that on the contralateral side is not shown. $C$, More opacification than that on the contralateral side. $D$, Exuberant.

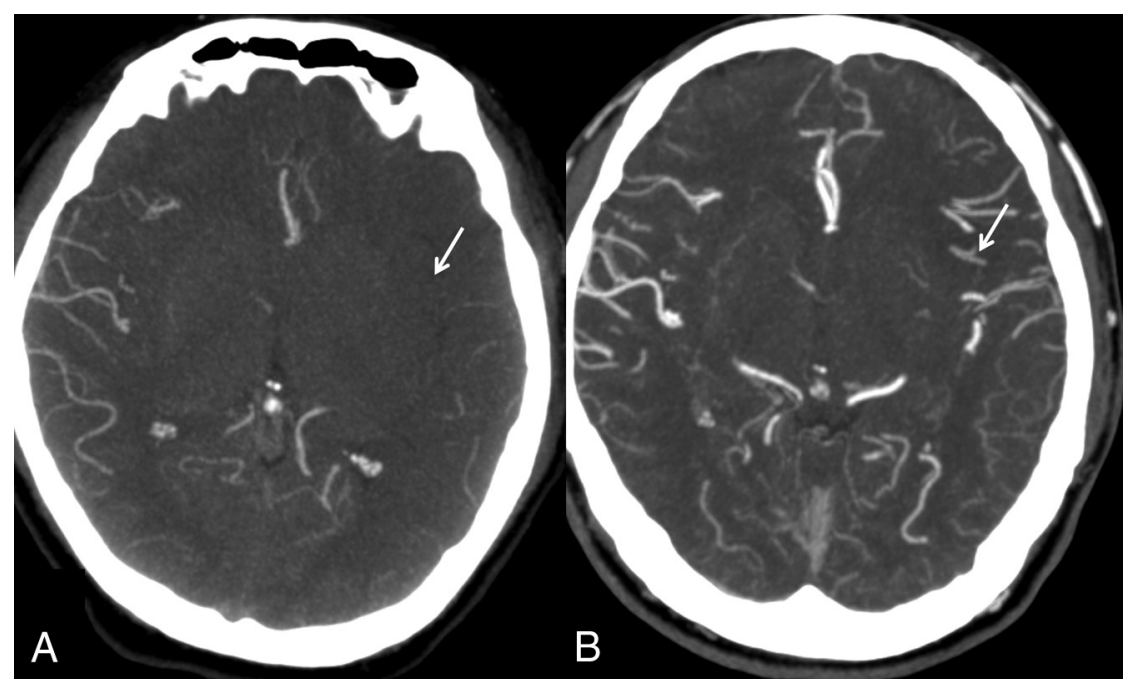

FIG 3. Modified Tan system. A, Less than $50 \%$ of the MCA territory. B, More than $50 \%$ of the MCA territory.

artery not seen; 1 , less prominent; 2, equal or more prominent compared with a matching region in the opposite hemisphere) in the 6 ASPECTS cortical regions (M1-6), the caudate, insular ribbon, internal capsule, and lentiform nucleus to form a score from 0 to 20 (Fig 4). ${ }^{16,17}$

\section{Statistical Methods}

We present the numeric variables as mean and SD or median and range. Categoric variables are presented as percentages. Numeric predictors were tested by using a 2-sample $t$ test or Mann-Whitney $U$ test when applicable. Categoric variables were evaluated by using the $\chi^{2}$ test or Fisher exact test when applicable. Variables found to have a significant association $(P<.05)$ were entered into the multivariable model to perform logistic regression for determining the independent predictors of the prespecified good and bad functional outcomes at 3 months. To improve the robustness of our statistical model, we also included variables with $P<.10$ into a multivariable logistic regression model with a backward stepwise selection procedure. Associations are presented as odds ratios with corresponding 95\% confidence intervals. Interobserver variability for the assessment of collateral status between the 2 observers was tested by using $\kappa$ statistics. Statistical analyses were performed by using the Statistical Package for Social Sciences, Version 20 (IBM, Armonk, New York).

\section{RESULTS}

In the study time, 2409 patients with strokes were seen, and of these, 200 patients satisfied the inclusion criteria for this study. The median age was 63 years (range, 33-92 years), and 31.5\% were women. The pre-tPA median NIHSS score was 19 points (range, 3-33). A relatively higher proportion $(73,36.5 \%)$ of patients had atrial fibrillation. Other factors are listed in Table 1. Overall, 93 (46.6\%) patients achieved good functional outcome at 3 months, and extremely poor outcomes were noted in 34 (17\%) patients (Fig 5). mRS $0-2$ at 3 months was achieved in 107 (53.5\%) patients.

The degree of agreement between the 2 independent neuroradiologists for interpreting the collaterals was best for the modified Tan system $(\kappa=0.93 ; 95 \% \mathrm{CI}$, $0.91-0.95)$. The level of agreement for the grading of collaterals by other systems was the following: Mass system $(\kappa=0.82$; 95\% CI, $0.75-0.84$ for leptomeningeal and $\kappa=$ $0.87 ; 95 \%$ CI, $0.80-0.91$ for Sylvian fissure vessels), Miteff system 
$(\kappa=0.91 ; 95 \%$ CI, $0.86-0.93)$, and ASPECTS-based grading system $(\kappa=0.77 ; 95 \%$ CI, $0.70-0.81)$.

On univariable analysis, younger age, female sex, hypertension, diabetes, atrial fibrillation, small infarct core on noncontrast CT (ASPECTS $\geq 8$ ), recanalization of the vessel, lower pre-tPA NIHSS scores, and good collaterals according to Tan methodology, ASPECTS methodology, and Miteff methodology were found to be significantly associated with good functional out-

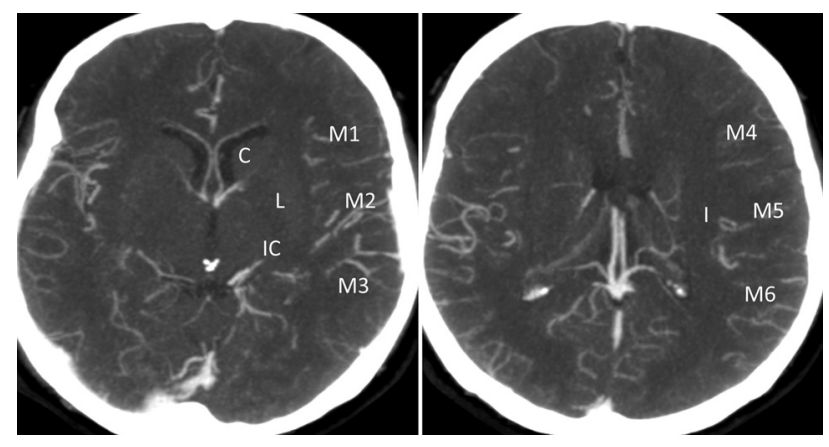

FIG 4. The ASPECTS system scores arteries distal to the occlusion (0, artery not seen; 1 , less prominent; 2 , equal or more prominent compared with a matching region in the opposite hemisphere) in the 6 ASPECTS cortical regions (M1-6), the caudate, insular ribbon, internal capsule, and lentiform nucleus to form a score from 0 to 20 .

Table 1: Baseline characteristics of the study population $(N=200)$

\begin{tabular}{lc}
\multicolumn{1}{c}{ Variables } & No. \\
\hline Median age (range) (yr) & $63(35-92)$ \\
Female sex (\%) & $63(31.5)$ \\
Hypertension (\%) & $131(65.5)$ \\
Diabetes (\%) & $60(30)$ \\
Dyslipidemia (\%) & $102(51)$ \\
Smoker (\%) & $57(28.5)$ \\
Atrial fibrillation (\%) & $73(36.5)$ \\
Pre-tPA systolic BP (range) & $152(110-215)$ \\
Pre-tPA NIHSS score (range) & $19(3-33)$ \\
Median onset to treatment (range) & $155(73-275)$ \\
TOAST criteria & \\
Large-artery atherosclerosis & $52(26 \%)$ \\
Cardioembolic & $96(48 \%)$ \\
Lacunar & $0(0 \%)$ \\
Undetermined cause & $42(21 \%)$ \\
Stroke of other etiology & $10(5 \%)$ \\
Location of occlusion & \\
Tandem & $6(3 \%)$ \\
Terminal ICA & $53(26.5 \%)$ \\
M1 & $76(38 \%)$ \\
Distal MCA & $65(32.5 \%)$ \\
Modified Rankin Scale 0-1 at 3 mo (\%) & $93(46.6)$ \\
Modified Rankin Scale 5-6 at 3 mo (\%) & $34(17)$ \\
\hline
\end{tabular}

Note:-TOAST indicates Trial of Org 10172 in Acute Stroke Treatment ${ }^{12}$; BP, blood pressure.

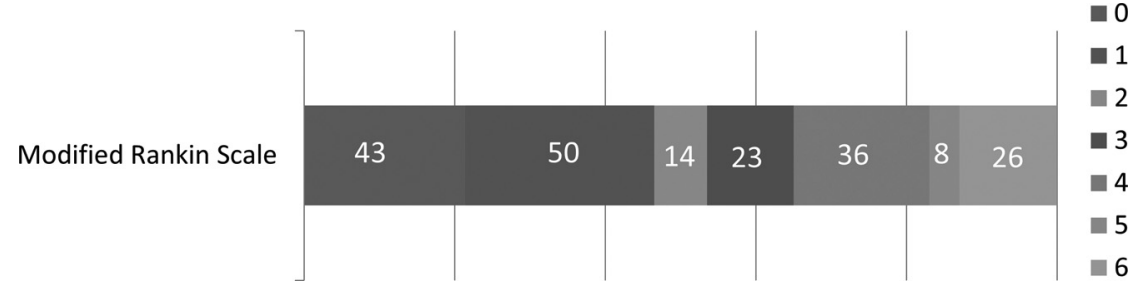

FIG 5. Three-month functional outcomes of patients by the modified Rankin Scale 0-6. comes (Table 2). On multivariable logistic regression, only lower NIHSS (OR, 1.186 per NIHSS point; 95\% CI, 1.079-1.302; $P=$ $.001)$, the presence of recanalization (OR, 5.599; 95\% CI, $1.560-$ 20.010; $P=.008$ ), and Miteff grading system for intracranial collaterals (OR, 3.341; 95\% CI, 1.241-8.996; $P=.017$ ) were found to independently predict good functional outcome. While modified Tan and Maas methodologies failed to predict good functional outcome, ASPECTS methodology showed a trend toward statistical significance (OR, 1.784 per point; 95\% CI, 0.820-9.761; $P=$ $.068)$. On ordinal regression analysis Miteff grading was still the most effective (On-line Table).

During the analyses for extremely poor outcome (mRS 5-6) at 3 months, the Miteff grading system with only distal superficial branches reconstituted (OR, 2.592; 95\% CI, 1.113-6.038; $P=$ $.027)$, the Maas grading system with absent collaterals on the affected side (OR, 2.580; 95\% CI, 1.075-6.187; $P=.034$ ), and ASPECTS methodology score $\leq 5$ points (OR, 2.685; 95\% CI, $1.156-$ $6.237 ; P=.022)$ were significantly associated with poor outcome at 3 months. Other significant cofactors were higher NIHSS score (OR, 1.143 per point; 95\% CI, 1.057-1.235; $P=.001$ ) and older age (OR, 1.040 per year increase; $95 \% \mathrm{CI}, 1.003-1.079 ; P=.034$ ). Symptomatic intracranial hemorrhage was observed in only 8 (4\%) patients, while $26(13 \%)$ patients died within the first 3 months after AAIS. None of the methods of collateral grading could predict mortality or symptomatic intracranial hemorrhage.

\section{DISCUSSION}

Our results show that the Miteff system of grading intracranial collaterals on CTA is the only method that could reliably predict good outcomes at 3 months in patients treated with IV-tPA. Extremely poor outcome was easier to predict by most (Maas, Miteff, and ASPECTS methodology) of the collateral grading systems.

Studies in mice with MCA occlusions have shown that infarction is significantly smaller if extensive collaterals develop. ${ }^{6}$ Similarly, in humans, the presence of robust collateral flow on conventional angiography is associated with smaller infarcts and better clinical outcome. ${ }^{18-21}$ The presence of effective collateral blood flow may influence the response to IV-tPA in AAIS by transporting fibrinolytics to both sides of the thrombus and facilitating its dissolution or limiting extension of the occlusion. $^{20,22-24}$ Furthermore, patients with good collaterals might carry a lower risk of hemorrhagic complications. ${ }^{13,25}$ Therefore, information about collateral flow in AAIS may help in establishing early prognosis and in planning various therapeutic approaches. For example, in patients with a higher risk of bleeding or more associated comorbidities, a good Mitteff score could lead the treating physician to initiate treatment with IV-tPA. The converse can also be true in patients with poor Mitteff scores, in whom one might withhold IVtPA treatment.

Previous studies have demonstrated an association between the degree of leptomeningeal collaterals and good outcome in patients not treated with IV-tPA or endovascular intervention. ${ }^{26}$ However, 
Table 2: Determinants of functional outcome in patients with thrombolyzed acute ischemic stroke

\begin{tabular}{|c|c|c|c|}
\hline Variable & $\begin{array}{c}\text { mRS 0-1 } \\
(n=93)\end{array}$ & $\begin{array}{c}\mathrm{mRS}-2-6 \\
(n=107)\end{array}$ & $P$ Value \\
\hline Median age (yr) (range) & $58(33-89)$ & $71(34-92)$ & .040 \\
\hline Female sex (\%) & $20(21.5)$ & $43(40.2)$ & .005 \\
\hline Hypertension (\%) & $51(54.8)$ & $80(74.8)$ & .034 \\
\hline Diabetes (\%) & $22(23.6)$ & $38(35.8)$ & .069 \\
\hline Dyslipidemia (\%) & $45(48.4)$ & $57(53.3)$ & .491 \\
\hline Atrial fibrillation (\%) & $23(24.7)$ & $50(46.9)$ & .001 \\
\hline Smoker (\%) & $33(35.5)$ & $24(22.4)$ & .119 \\
\hline Median NIHSS pre-tPA (range) & $14(3-29)$ & $21(8-33)$ & .477 \\
\hline Mean SBP pre-tPA (range) & $142(110-209)$ & $154(110-210)$ & .777 \\
\hline Median onset-to-treatment time (range) & $152(91-275)$ & $158(73-265)$ & 199 \\
\hline ASPECTS $<7$ & $36(38.7)$ & $62(57.9)$ & .010 \\
\hline Recanalization of vessel & $70(75.3)$ & $76(71)$ & .040 \\
\hline Good collaterals by Tan method & $74(79.6)$ & $49(45.8)$ & .001 \\
\hline Good collaterals by Miteff method & $25(26.9)$ & $66(61.7)$ & .001 \\
\hline Good collaterals by Maas method & $42(45.2)$ & $23(21.5)$ & .005 \\
\hline Median score by ASPECTS methodology (range) & $11(0-18)$ & $8(1-18)$ & .021 \\
\hline
\end{tabular}

Note:-SBP indicates systolic blood pressure.

Rosenthal et $\mathrm{al}^{27}$ reported that leptomeningeal collaterals showed only a minimal positive impact in patients who did not achieve arterial recanalization, and they did not show any impact if complete recanalization of the occluded intracranial artery was achieved. We hypothesized that a favorable pattern of leptomeningeal collaterals on the pre-tPA CTA would result in improved functional outcome. We evaluated the 4 most accepted methodologies to test our hypothesis and to identify the best collateral grading system for predicting outcome in our patients treated with IV-tPA.

Being a simple grading system, the modified Tan system is easily reproducible among readers. ${ }^{15}$ However, it was not found to be a useful predictor of good outcome. Similarly, the ASPECTS-based grading system and the Maas system failed to predict good functional outcome in our patients with thrombolysed anterior circulation. Only the Miteff grading system could determine good functional outcomes at 3 months. Perhaps, this result is due to the focus on the Sylvian fissure, the insular region that is known to predict the penumbral mismatch in acute MCA infarcts. ${ }^{28}$ Good collaterals in these anatomic regions improve perfusion, reduce final infarct size, and lead to better outcomes. Focus on the easily recognizable landmarks (Sylvian fissure and insula) and a high rate of reproducibility are the main strengths of the Miteff scoring system and enable it to be a reliable predictor of good outcome in patients with AAIS treated with IV-tPA.

Conventional angiography is considered the criterion standard for collateral-flow assessment, but its invasive nature limits its use in clinical practice. Noninvasive and safer methods, including transcranial Doppler and MR angiography, can evaluate larger arteries such as the circle of Willis. However, they do not have enough resolution to evaluate the leptomeningeal vascular bed. ${ }^{6}$ CTA is widely available and has a higher degree of spatial resolution, which enables the assessment of leptomeningeal collaterals. Furthermore, CTA can be performed with concurrent imaging of the cervical arteries and CT perfusion for the "multimodal" penumbral assessment. ${ }^{29,30}$ Despite these advantages, only a few studies have used CTA to assess the degree of collateral circulation in AAIS. ${ }^{6,27}$ With rapid advancements in technology, better methods for collateral assessment are evolving. Perhaps, a multiphasic
CTA that provides information about early and late arterial phases may serve as a better tool for the evaluation of intracranial collaterals in acute ischemic stroke and may serve as a surrogate for perfusion studies, eventually becoming a part of standard routine reporting of CTAs in patients with acute ischemic stroke. However, multiphasic CTA would need to be evaluated and validated in a larger prospective study.

Some limitations of our study need to be acknowledged. First, although we evaluated a moderate-sized cohort of 200 patients, this sample size may not be sufficient to analyze the subtle differences among various collateral grading systems. Second, despite their common aim of predicting the prognosis, various grading systems have their own inherent limitations. For example, the Maas system is less accurate in patients with a previous intracranial occlusion due to a limited side-to-side comparison. This finding may be specifically relevant to our Asian cohort of patients with AAIS with a high prevalence of intracranial stenosis. ${ }^{31}$ Similarly, in the ASPECTS grading system, basal ganglia regions have less vasculature and result in a higher weighting for leptomeningeal vessels. The location of the occlusion may also affect the predictive ability of the collateral scoring system. Third, despite its high spatial resolution, CTA does not provide enough information about flow dynamics. Therefore, CTA may lead to an overestimation of the degree of collateral circulation. ${ }^{32}$ Perhaps, consideration of internal cerebral vein asymmetry in patients with severe occlusive disease of the ICA or MCA may add significant hemodynamic information to the preferred method of scoring the intracranial collaterals in AAIS. ${ }^{33}$ Fourth, the retrospective design of our study could be a limitation. However, the collateralgrading readers were blinded to the clinical information and outcome. Fifth, as evident from a high NIHSS score at presentation (median, 19 points), our study included a larger proportion of patients with severe AAIS. Finally, none of the methods of collateral grading were associated with early mortality or symptomatic intracranial hemorrhage. We suspect that this observation is related to the sample size of our study, and a larger study is recommended to evaluate this association, especially between intracranial collateral grading and symptomatic intracranial hemorrhage. ${ }^{25}$

\section{CONCLUSIONS}

Our study provides a comparative assessment of various grading methods for intracranial collaterals for establishing an early prognosis in patients with AAIS treated with IV-tPA. Only the Miteff system was found to be a reliable predictor of favorable outcome in IV-treated patients with AAIS. We believe that routine grading and reporting of intracranial collaterals will rapidly become a part of the standard stroke CTA reports as the clinical relevance becomes more and more apparent. Further larger scale prospective studies, with evaluation of additional information about cerebral 
hemodynamics, are recommended to develop better grading criteria for intracranial collaterals in AAIS.

Disclosures: Leonard Yeo—RELATED: Grant: National Medical Research Council Singapore, * Comments: grant No. CNIG12nov001. Benjamin Wakerley-UNRELATED: Travel/Accommodations/Meeting Expenses Unrelated to Activities Listed: Novartis, Comments: sponsored for the 2014 American Academy of Neurology meeting. *Money paid to the institution.

\section{REFERENCES}

1. Thomassen L, Waje-Andreassen U, Naess H, et al. Doppler ultrasound and clinical findings in patients with acute ischemic stroke treated with intravenous thrombolysis. Eur J Neurol 2005;12: 462-65

2. Rha JH, Saver JL. The impact of re-canalization on ischemic stroke outcome: a meta-analysis. Stroke 2007;38:967-73

3. Liebeskind DS, Kim D, Changizi K, et al. Collateral failure? Late mechanical thrombectomy after failed intravenous thrombolysis. J Neuroimaging 2008;20:78-82

4. Liebeskind D. Collaterals in acute stroke: beyond the clot. Neuroimaging Clin N Am 2005;15:553-73

5. Liebeskind DS. Understanding blood flow: the other side of an acute arterial occlusion. Int J Stroke 2007;2:118-20

6. Liebeskind DS. Collateral circulation. Stroke 2003;34:2279-84

7. Liebeskind DS. Neuroprotection from the collateral perspective. IDrugs 2005;8:222-28

8. Sakoh M, Ostergaard L, Gjedde A, et al. Prediction of tissue survival after middle cerebral artery occlusion based on changes in the apparent diffusion of water. J Neurosurg 2001;95:450-58

9. Sobesky J, Weber OZ, Lehnhardt FG, et al. Which time-to-peak threshold best identifies penumbral flow? A comparison of perfusion-weighted magnetic resonance imaging and positron emission tomography in acute ischemic stroke. Stroke 2004;35:2843-47

10. Lin CS, Polsky K, Nadler JV, et al. Selective neocortical and thalamic cell death in the gerbil after transient ischemia. Neuroscience 1990;35:289-99

11. Jauch EC, Saver JL, Adams HP Jr, et al. Guidelines for the early management of patients with acute ischemic stroke: a guideline for healthcare professionals from the American Heart Association/ American Stroke Association. Stroke 2013;44:870-947

12. Adams HP Jr, Bendixen BH, Kappelle LJ, et al. Classification of subtype of acute ischemic stroke: definitions for use in a multicenter clinical trial: TOAST_-Trial of Org 10172 in Acute Stroke Treatment. Stroke 1993;24:35-41

13. Miteff F, Levi CR, Bateman GA, et al. The independent predictive utility of computed tomography angiography collateral status in acute ischemic stroke. Brain 2009;132:2231-38

14. Maas MB, Lev MH, Ay H, et al. Collateral vessels on CT angiography predict outcome in acute ischemic stroke. Stroke 2009;40:3001-05

15. Tan IY, Demchuk AM, Hopyan J, et al. CT angiography clot burden score and collateral score: correlation with clinical and radiologic outcomes in acute middle cerebral artery infarct. AJNR Am J Neuroradiol 2009;30:525-31

16. Menon BK, Smith EE, Modi J, et al. Regional leptomeningeal score on CT angiography predicts clinical and imaging outcomes in patients with acute anterior circulation occlusions. AJNR Am J Neuroradiol 2011;32:1640-45

17. Choi JY, Kim EJ, Hong JM, et al. Conventional enhancement CT: a valuable tool for evaluating pial collateral flow in acute ischemic stroke. Cerebrovasc Dis 2011;31:346-52

18. Bozzao L, Fantozzi LM, Bastianello S, et al. Early collateral blood supply and late parenchymal brain damage in patients with middle cerebral artery occlusion. Stroke 1989;20:735-40

19. von Kummer R, Holle R, Rosin L, et al. Does arterial recanalization improve outcome in carotid territory stroke? Stroke 1995;26:581-87

20. Ringelstein EB, Biniek R, Weiller C, et al. Type and extent of hemispheric brain infarctions and clinical outcome in early and delayed middle cerebral artery recanalization. Neurology 1992;42:289-98

21. Mohammad YM, Christoforidis GA, Bourekas EC, et al. Qureshi grading scheme predicts subsequent volume of brain infarction following intra-arterial thrombolysis in patients with acute anterior circulation ischemic stroke. J Neuroimaging 2008;18:262-67

22. Jo KD, Saver JL, Starkman S, et al. Predictors of recanalization with mechanical thrombectomy for acute ischemic stroke (abstract). Stroke 2008;39:599

23. Nogueira RG, Liebeskind DS, Sung G, et al. Predictors of good clinical outcomes, mortality, and successful revascularization in patients with acute ischemic stroke undergoing thrombectomy: pooled analysis of the Mechanical Embolus Removal in Cerebral Ischemia (MERCI) and Multi MERCI trials. Stroke 2009;40:3777-83

24. Jovin TG, Gupta R, Horowitz MB, et al. Pretreatment ipsilateral regional cortical blood flow influences vessel recanalization in intra-arterial thrombolysis for MCA occlusion. AJNR Am J Neuroradiol 2007;28:164-67

25. Bang OY, Saver JL, Kim SJ, et al. Collateral flow averts hemorrhagic transformation after endovascular therapy for acute ischemic stroke. Stroke 2011;42:2235-39

26. Lima FO, Furie KL, Silva GS, et al. The pattern of leptomeningeal collaterals on CT angiography is a strong predictor of long-term functional outcome in stroke patients with large vessel intracranial occlusion. Stroke 2010;41:2316-22

27. Rosenthal ES, Schwamm LH, Roccatagliata L, et al. Role of recanalization in acute stroke outcome: rationale for a $\mathrm{CT}$ angiogram-based "Benefit of Recanalization" model. AJNR Am J Neuroradiol 2008; 29:1471-75

28. Kamalian S, Kemmling A, Borgie RC, et al. Admission insular infarction $>25 \%$ is the strongest predictor of large mismatch loss in proximal middle cerebral artery stroke. Stroke 2013;44:3084-89

29. Zhao L, Barlinn K, Bag AK, et al. Computed tomography perfusion prognostic maps do not predict reversible and irreversible neurological dysfunction following reperfusion therapies. Int J Stroke 2011;6:544-46

30. Wintermark M, Meuli R, Browaeys P, et al. Comparison of CT perfusion and angiography and MRI in selecting stroke patients for acute treatment. Neurology 2007;68:694-97

31. De Silva DA, Woon FP, Pin LM, et al. Intracranial large artery disease among OCSP subtypes in ethnic South Asian ischemic stroke patients. J Neurol Sci 2007;260:147-49

32. Arnold M, Nedeltchev K, Mattle HP, et al. Intra-arterial thrombolysis in 24 consecutive patients with internal carotid artery occlusions. J Neurol Neurosurg Psychiatry 2003;74:739-42

33. Sharma VK, Yeo LL, Teoh HL, et al. Internal cerebral vein asymmetry on follow-up brain computed tomography after intravenous thrombolysis in acute anterior circulation ischemic stroke is associated with poor outcome. J Stroke Cerebrovasc Dis 2014;23:e39-45 
P lease note that there is an error in Table 2 of the February 2015 article "Assessment of Intracranial Collaterals on CT Angiography in Anterior Circulation Acute Ischemic Stroke" by Yeo et al (AJNR Am J Neuroradiol 2015;36:289-94, originally published on-line on October 16, 2014, doi:10.3174/ajnr.A4117). The population of patients with good collaterals by Miteff scoring with good outcomes has been switched with the population with poor outcomes. The corrected Table 2 is reproduced below with the corrected segment shown in bold. The authors apologize for the error.

http://dx.doi.org/10.3174/ajnr.A4381

Table 2: Determinants of functional outcome in patients with thrombolyzed acute ischemic stroke

\begin{tabular}{|c|c|c|c|}
\hline Variable & $\begin{array}{c}\text { mRS 0-1 } \\
(n=93)\end{array}$ & $\begin{array}{c}\text { mRS 2-6 } \\
(n=107)\end{array}$ & $P$ Value \\
\hline Median age (yr) (range) & $58(33-89)$ & $71(34-92)$ & .040 \\
\hline Female sex (\%) & $20(21.5)$ & $43(40.2)$ & .005 \\
\hline Hypertension (\%) & $51(54.8)$ & $80(74.8)$ & .034 \\
\hline Diabetes (\%) & $22(23.6)$ & $38(35.8)$ & .069 \\
\hline Dyslipidemia (\%) & $45(48.4)$ & $57(53.3)$ & .491 \\
\hline Atrial fibrillation (\%) & $23(24.7)$ & $50(46.9)$ & .001 \\
\hline Smoker (\%) & $33(35.5)$ & $24(22.4)$ & .119 \\
\hline Median NIHSS pre-tPA (range) & $14(3-29)$ & $21(8-33)$ & .477 \\
\hline Mean SBP pre-tPA (range) & $142(110-209)$ & $154(110-210)$ & .777 \\
\hline Median onset-to-treatment time (range) & $152(91-275)$ & $158(73-265)$ & 199 \\
\hline ASPECTS $<7$ & $36(38.7)$ & $62(57.9)$ & .010 \\
\hline Recanalization of vessel & $70(75.3)$ & $76(71)$ & .040 \\
\hline Good collaterals by Tan method & $74(79.6)$ & $49(45.8)$ & .001 \\
\hline Good collaterals by Miteff method & $66(61.7)$ & $25(26.9)$ & .001 \\
\hline Good collaterals by Maas method & $42(45.2)$ & $23(21.5)$ & .005 \\
\hline Median score by ASPECTS methodology (range) & $11(0-18)$ & $8(1-18)$ & .021 \\
\hline
\end{tabular}

Note:-SBP indicates systolic blood pressure. 